\title{
AvoIDING SHIP-INDUCED LIGHT-FIELD Perturbation in the Determination of Oceanic Optical Properties
}

By Kirk J. Waters,

Raymond C. Smith and Marlon R. Lewis

$\mathrm{T}$ LHE DETERMINATION of in-water spectral irradiance and the subsequent estimation of ocean optical properties is essential for the meaningful understanding and predictive modeling of the marine photoenvironment; this in turn is fundamental to the understanding of both biological and physical oceanographic processes. Further, the accurate estimation of near-surface optical properties is essential for the surface validation of ocean color satellite sensors. Twoprimary sources of error in the determination of optical properties at sea are the perturbation of the in-water radiant energy field due to the ship (Gordon, 1985; Smith and Baker, 1986) and the estimation of quantitative corrections for atmospheric variability in radiant energy incident on the sea-surface during in-water measurements (Smith and Baker, 1984). Here we briefly describe two new optical oceanographic instrument and deployment techniques designed to reduce or eliminate these sources of error. The first is an Optical Free Fall Instrument (OFFI) designed to profile the water column away from potential perturbation effects of the ship: the second is an Optical Surface Floating Instrument (OSFI) designed to obtain continuous optical data just below the air-water interface. A cartoon illustrating deployment of these instruments is shown in Fig. 1.

Shipboard observations of spectral irradiance and radiance as a function of depth in the water column are commonly obtained by lowering an instrument on a hydrowire on the "sunny side" of the ship. Typically the instrument is lowered relatively close to the ship, which results in significant perturbation of the measured in-water light field. Using Monte Carlo simulations, Gordon (1985) has shown that the bias in determining downwelling irradiance seldom exceeds $1-2 \%$ for clear skies with the sun within $\pm 45^{\circ}$ of the beam of the ship, while the error can exceed $10 \%$ if the sun is off the bow or stern. While generally small, this error under clear skies is a function of wavelength. The error for overcast skies with a diffuse light field will be increased by the fraction of the sky obscured by the ship, after accounting for refraction and the Fresnel

Kirk J. Waters and Raymond C. Smith, University of California, Santa Barbara. CSL/Center for Remote Sensing and Environmental Optics. Santa Barbara, CA 93106; Marlon R. Lewis. Department of Oceanography, Dalhousie University, Halifax. Nova Scotia, Canada, B3H $4 \mathrm{~J} 1$ reflection at the air-water interface, and can be as much as $20-30 \%$. This error shows relatively little wavelength dependence. These perturbation errors are largest in the top attenuation length of the ocean, the region where maximum accuracy is desired for both remote sensing applications and bio-optical modeling.

Determinations of upwelling radiance are especially important in the development of satellite algorithms for the remote estimation of chlorophyll concentration. The error in determining upwelling radiance varies between $10-20 \%$ unless the instrument can be 5 $10 \mathrm{~m}$ from the side of the ship. Voss et al. (1986) have shown this error as a function of distance from the ship from $0-9 \mathrm{~m}$ and found up to a $40 \%$ error for overcast conditions. As a consequence of the errors caused by ship perturbation of the light field, optical instruments have been floated a considerable distance from the ship in order to obtain accurate data for satellite algorithms (Gordon and Clark, 1980; Clark, 1981). However, constraints on ship-time and unfavorable sea-states often prevent or restrict use of this procedure. Further, at-sea efforts in support of satellite observations frequently necessitate synoptic shipboard sampling, which requires the rapid deployment and recovery of optical instruments (Smith et al., 1987b). To overcome these limitations, we have developed a free-fall instrument and deployment technique to avoid both the light field perturbation by the ship and to allow rapid deployment.

The OFFI is a modification of the MER-2020 underwater units described by Booth and Smith (1988) with the free-fall technology based on a prototype instrument with a single optical band(Lewis etal., 1986). The underwater units include sensors for determination of depth, upwelling radiance $\left(\mathrm{L}_{4}\right.$ at $410,441,488,520$, $683 \mathrm{~nm})$, downwelling irradiance $\left(E_{d}\right.$ at $410,441,488$, $520,560 \mathrm{~nm}$ ), temperature, and two axis tilt and roll. Bandwidths of the optical sensors are $10 \mathrm{~nm}$ full-width at half-maximum. A deck-based sensor suite, operated concurrently with vertical casts of the OFFI, provides a determination of downwelling spectral irradiance above the surface, $\mathrm{E}_{d}\left(0^{+}, \lambda\right)$, at the same wavelengths as the in-water unit. The OFFI is contained in a $75 \mathrm{~cm}$ long $x 20 \mathrm{~cm}$ diameter aluminum case with the irradiance sensors at the top (to determine downwelling spectral irradiance $E_{d}(z, \lambda)$ ) and the radiance sensors at the bottom (to determine upwelling spectral radiance $\mathrm{L}_{u}(\mathrm{z}, \lambda)$ ). The case is outfitted with buoyant fins to give it stability and 
control the descent rate. The OFFI can be programmed for various sampling rates and logs data internally, first in memory, then on tape. A conducting cable makes it possible to transmit data directly to the ship in realtime. For free-fall vertical casts, the OFFI was programmed to sample as rapidly as possible, two to three samples per second, giving six to eight samples per meter during descent.

The system was first tested from February 17 through March 15, 1988, in the equatorial Pacific on the R/V Wecoma and then tested with a comparable instrument from July 20 through August 8, 1988, in the Santa Barbara Channel on the R/V Melville. Free-fall vertical casts were deployed using a $0.63 \mathrm{~cm}$ Kevlar-strength member conducting cable (Courtland) and a "fish out" technique. The Kevlar cable was chosen for its combination of strength and near-neutral buoyancy. The "fish out" technique requires letting the instrument free fall from the side of the ship while letting the ship either drift or maneuver away from the entry location. After sufficient distance between the OFFI and ship is obtained. the OFFI is raised and typically surfaces at distances greater than $20 \mathrm{~m}$ from the ship. The fins are critical in this technique as they allow the OFFI to kite away from the ship during ascent. After fishing the unit out far enough to reduce the effects of ship perturbation to the light field, the OFFI unit is allowed to free fall for a vertical cast. While profiling, the cable is hand-fed into the water to ensure slack cable in the water. This minimizes the cable pull on the OFFI and permits the instrument to maintain a vertical orientation during descent. During a deployment, it was possible to do as many as five to six vertical casts in one half-hour. The distance from the ship increases with subsequent profiles as the ship continues to drift. This can result in vertical profiles obtained at distances of over $100 \mathrm{~m}$ from the ship, distances which are completely clear of ship perturbation effects. As no method was available to accurately estimate the distance from the ship, no comparison as a function of distance was made.

Compared to typical hydrowire deployed instruments (i.e., the Bio-Optical Profiling System (BOPS), (Smith et al., 1984)), the OFFI has several advantages in addition to the avoidance of ship shadow. Typically, an in-water optical instrument is subject to the vagaries of surface motion transmitted via the hydrowire. Because it is not subject to this interference, the OFFI

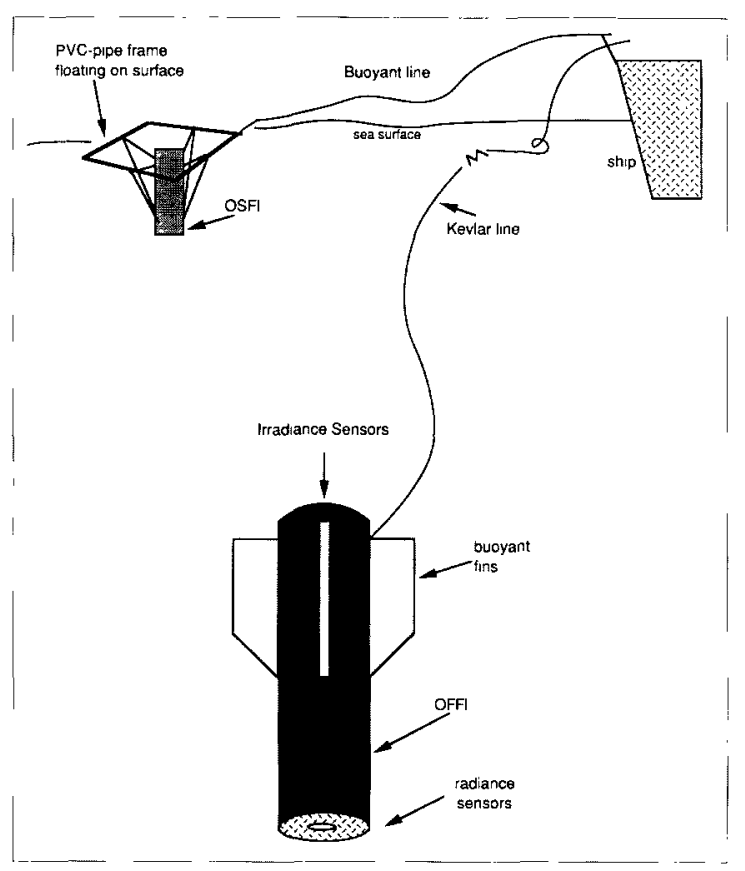

Fig. I: Cartoon of OFFI and OSFI instruments as deployed.

makes a smoother descent with more uniform sampling at regular depth intervals. This allows for a more rapid descent, reducing sky variability and the need for smoothing procedures in data analysis (Smith and Baker, 1984). The faster descent rate also allows multiple profiles in a short time period from a single deployment over the side of the ship.

Figure 2 (p. 20) shows one channel of spectral irradiance $(441 \mathrm{~nm})$ collected under fully overcast skies for the OFFI. The inset displays near surface irradiance data in detail and shows the variability caused by refraction of the optical signal by surface waves. Near-surface spectral irradiance data under cloudy skies are maximum perturbation conditions for a conventionally deployed optical sensor. The OFFI data are independent of ship perturbation effects. This can be demonstrated by comparing the diffuse attenuation coefficient for irradiance, $\mathrm{K}_{\mathrm{d}}(\mathrm{z}, \lambda)$, with chlorophyll fluorescence as determined with a nearly contemporaneous BOPS cast. For ocean waters, where 


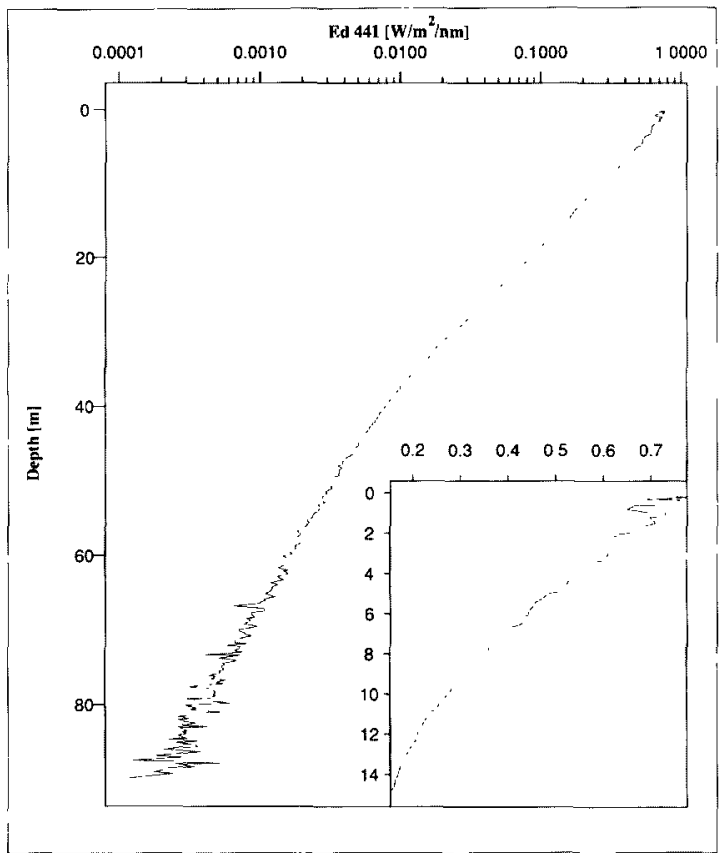

Fig. 2: OFFI $E_{d}(441 \mathrm{~nm})$ vs depth with inset for top $20 \mathrm{~m}$.

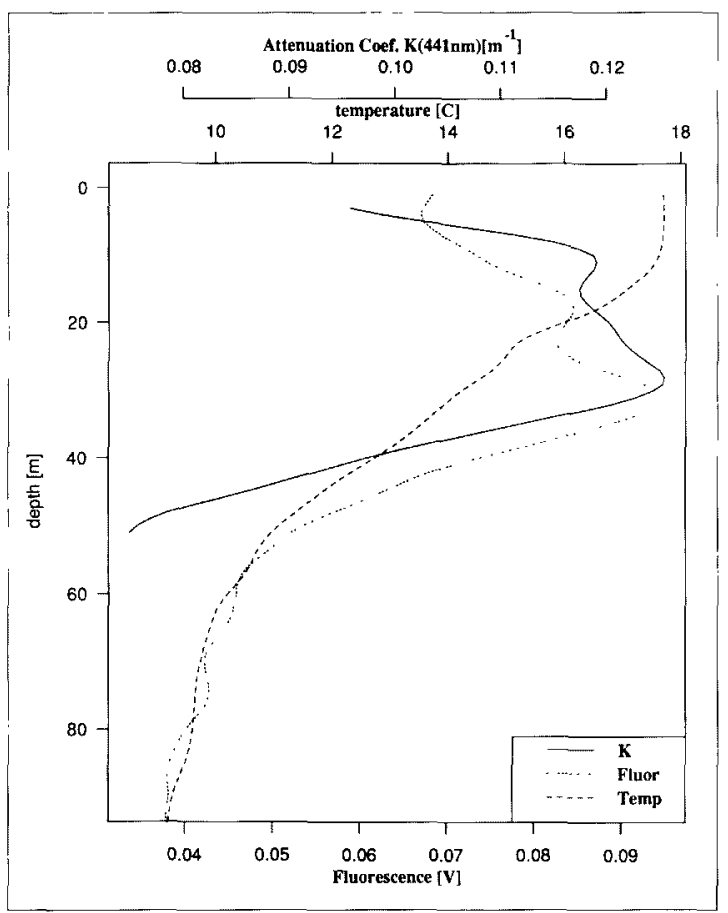

Fig. 3: Fluorometer (dotted) and temperature (solid) records taken concurrently with BOPS cast for the overcast day compared with $K(441 \mathrm{~nm})$.

the ratio of scattering to absorption is small, the attenuation coefficient is proportional to absorption of the dissolved and suspended constituents in the water column (Preisendorfer, 1961). Chlorophyll- $a$ is expected to be a dominant absorber at $441 \mathrm{~nm}$ so the depth profiles of $\mathrm{K}_{d}(\mathrm{z}, 441 \mathrm{~nm})$ and chlorophyll fluorescence (via fluorometer) should be similar as shown in Fig. 3 . It should be noted that near-surface $K$ values are particularly sensitive to perturbation in the light field (Smith and Baker, 1984, 1986) so that the relative similarity in vertical shape is especially meaningful. In particular, the near-surface $K$ values derived from the OFFI correspond well with the chlorophyll fluorescence determined in the upper mixed layer. A vertical offset between $K$ values and fluorescence values of approximately $10 \mathrm{~m}$ is reasonable when considering the time delay between OFFI and BOPS casts (about forty minutes) and the imperfect analogy between stimulated fluorescence and $\mathrm{K}$. As a consequence, we conclude that the near-surface behavior of $K_{\text {OFFI }}$ is consistent with the ancillary biological and physical properties of the water column.

In order to obtain shipboard estimates of $\mathbf{K}$ and other optical properties over large areas rapidly, it is necessary to make optical measurements quickly in all environmental conditions. Smith and Baker (1986) have described methods of ocean optical data analysis and experimental and theoretical techniques for correcting in-water optical data for atmospheric variability and changes in solar elevation. These techniques make use of above-water spectral irradiance data, $\operatorname{Ed}\left(0^{+}, \lambda\right)$, and theory to compute the irradiance just below the airwater interface, $\mathrm{E}_{d}\left(0^{-}, \lambda\right) . \mathrm{E}_{\mathrm{d}}\left(0^{-}, \lambda\right)$ is then used as a normalization factor to correct for variations in irradiance during a vertical profile or over the period of a day for a sequence of profiles. Actual determination of $\mathrm{E}_{d}\left(0^{-}, \lambda\right)$ is a more direct way to obtain this information.

An Optical Surface Floating Instrument (OSFI) was designed and built to maintain an MER-2020 unit $10-20 \mathrm{~cm}$ below the surface. The subsurface floater frame was designed so that it remained out of the field of view for solar zenith angles less than $80^{\circ}$. The OSFI consisted of a five foot square of 2 " polyvinylchloride (PVC) pipe attached to the MER-2020 with $3 / 8$ " wire cable and tethered to the ship by a $50 \mathrm{~m}$ line. The frame is quite durable; it withstood towing at one knot and had little difficulty withstanding three-meter swells. Deployment of this instrument permitted the continuous determination of both $\mathrm{E}_{d}\left(0^{-}, \lambda\right)$ and $\mathrm{L}_{\mathrm{u}}\left(0^{-}, \lambda\right)$ away from the influence of the ship. An example of OSFI data with one-minute samples taken on the same day as the overcast profiles is shown in Fig. 4. The one-minute samples are averages of 120 scans. The dashed vertical lines in Fig. 4 indicate when profiles were taken.

The $\mathrm{E}_{\mathrm{d}}\left(0^{-}, \lambda\right)$ determination can be used for correction of profiles if the sky conditions are variable during the profiling. Generally, a deck unit is used to collect above-surface data, which is then converted by an empirical algorithm to account for transmission loss through the air-water interface (Gordon, 1985; Smith and Baker, 1986). Also, it is notoriously difficult to mount optical sensors on ship superstructures so as to avoid reflecting surfaces, shadows from masts, stays and stack exhaust. The floater unit eliminates the need for such an algorithm and does not have the problems of ship perturbation, which may affect poorly located deck units. Examples of bio-optical analysis making use of $\mathrm{E}_{\mathrm{d}}(0, \lambda)$ data can be found in Smith et al. (1987a, 1989) and Bidigare et al. (1987). 


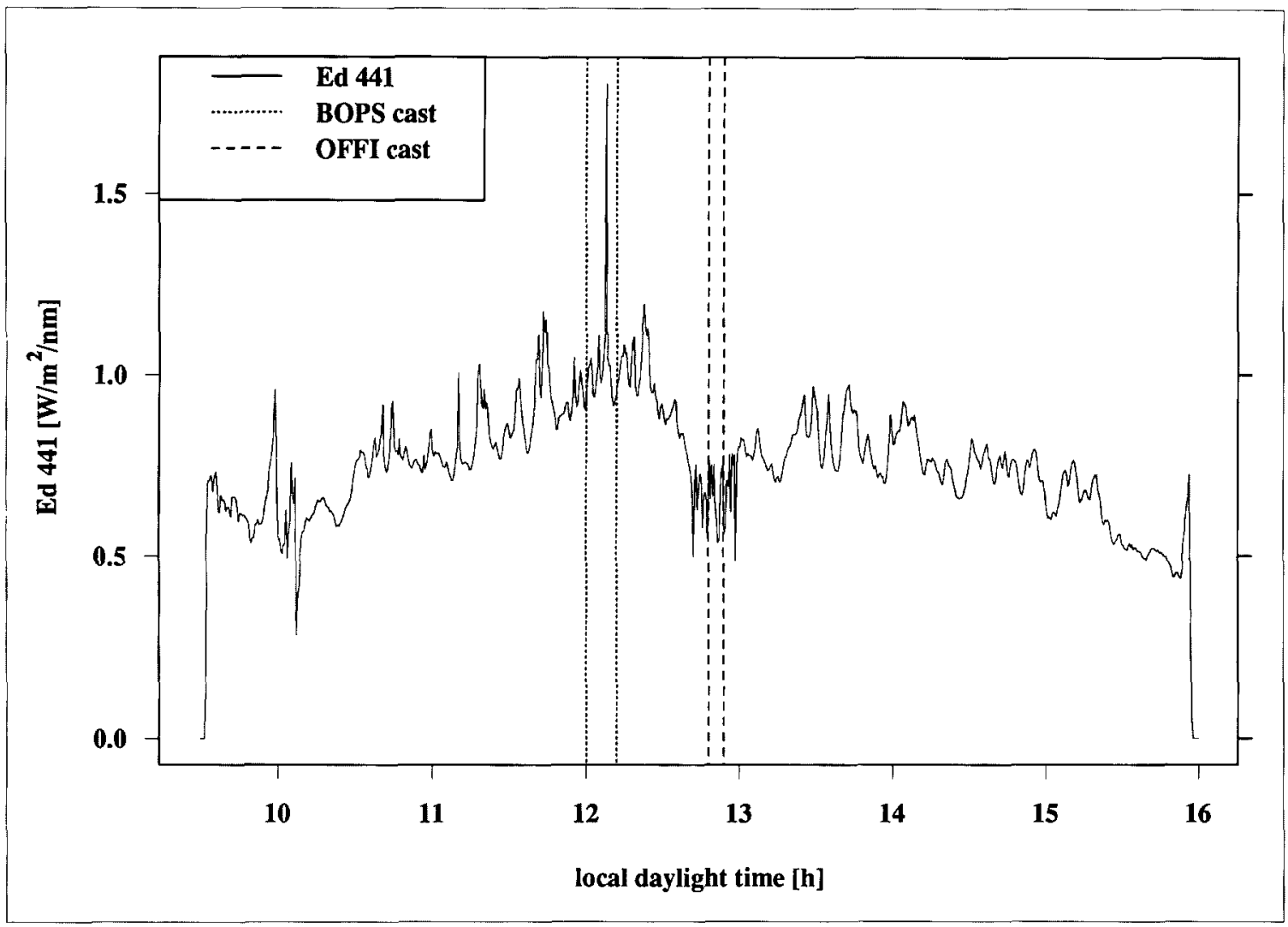

Fig. 4: Example of $O S F I E_{d}\left(0^{-}, \lambda\right)$ for 441 nm taken on the overcast day (August 3, 1988). Vertical dashed lines indicate the time span during which the two profiles were taken.

The Optical Free Fall Instrument permits in-water optical data to be obtained rapidly, relatively easily and in a way that avoids errors due to ship perturbation of the light field. While by no means replacing hydrowire instruments, it does provide more accurate light field data during unfavorable conditions. The Optical Surface Floater Instrument (OSFI) allows $\mathrm{E}_{d}\left(0^{-}, \lambda\right)$ to be continuously determined. These data are valuable for correcting in-water vertical profile data for light field variability caused by changes in atmospheric conditions and sun elevation. In addition, these data are those needed for surface validation of satellite ocean color sensors.

\section{References}

Bidigare, R.R., R.C. Smith, K.S. Baker and J. Marra, 1987: Oceanic primary production estimates from measurements of spectral irradiance and pigment concentrations. Global Biogeochemical Cycles, 1, 171-186.

Booth, C.R. and R.C. Smith, 1988: Moorable spectroradiometer in the Biowatt Experiment. Proceedings of The Society of Photo-Optical Instrumentation Engineers. Ocean Optics IX, 925, 176-188.

Clark, D.K., 1981: Phytoplankton pigment algorithms for the Nimbus-7 CZCS. In: Oceanography from Space. J.F.R. Gower, ed., Plenum, New York, 227-237.

Gordon, H.R., 1985: Ship perturbation of irradiance measurements at sea. 1: Monte Carlo simulations. Appl. Opt., 24, 41724182.

and D.K. Clark, 1980: Atmospheric effects in the remote sensing of phytoplankton pigments. Boundary Layer Meterol., 18, 299-313.
Lewis, M.R., W.G. Harrison, N.S. Oakey, D. Herbert and T. Platt, 1986: Vertical Nitrate Fluxes in the Oligotrophic Ocean. Science, 234, 870-873.

Preisendorfer, R.W., 1961: Theoretical proof of the existence of characteristic diffuse light in natural waters. Union of Geog. Geophys. Inst. Monogr., 10, 11-30.

, 1976: Hydrologic Optics, vol 1. Washington: U.S. Department of Commerce, 218 pp.

Smith, R.C. and K.S. Baker, 1984: The analysis of ocean optical data. Proceedings of The Society of Photo-Optical Instrumentation Engineers. Ocean Optics VII, 489, 119 126.

C.R. Booth and J.L. Star, 1984: Oceanographic Biooptical profiling system. Appl. Opt., 23, 2791. and K.S. Baker, 1986: The analysis of ocean optical data, II. Proceedings of The Society of Photo-Optical Instrumentation Engineers. Ocean Optics VIII,637,95-107.

R.R. Bidigare, B.B. Prézelin, K.S. Baker and J.M. Brooks, 1987a: Optical characterization of primary productivity across a coastal front. Mar. Biol.,96,575-591.

O.B. Brown, F.E. Hoge, K.S. Baker, R.H. Evans, R.N. Swift and W.E. Esaias, 1987b: Multiplatform sampling (ship, aircraft, and satellite) of a Gulf Stream warm core ring. Appl. Opt., 26, 2068-2081.

, B.B. Prézelin, R.R. Bidigare and K.S. Baker, 1989: Biooptical modeling of photosynthetic production in coastal waters. Limnol. Oceanogr., 34(8), 1526-1546.

Voss, K.J., J.W. Nolten and G.D. Edwards, 1986: Ship Shadow Effects on Apparent Optical Properties. Proceedings of The Society of Photo-Optical Instrumentation Engineers. Ocean Optics VIII, 637, 186-190. 口 